\title{
Wu Association Between Patient-Centered Medical Home Capabilities and Outcomes for Medicare Beneficiaries Seeking Care from Federally Qualified Health Centers
}

\author{
Justin W. Timbie, PhD' ${ }^{7}$, Peter S. Hussey, PhD', Claude M Setodji, PhD³, Amii Kress, PhD, MPH', \\ Rosalie Malsberger, MS², Tara A. Lavelle, PhD ${ }^{4}$, Mark W. Friedberg, MD, MPP2,5,6, \\ Suzanne G. Wensky, $P h D^{7}$, Katherine D. Giuriceo, $P h D^{7}$, and Katherine L. Kahn, $M D^{8,9}$
}

${ }^{1}$ RAND Corporation, Arlington, VA, USA; ${ }^{2}$ RAND Corporation, Boston, MA, USA; ${ }^{3}$ RAND Corporation, Pittsburgh, PA, USA; ${ }^{4}$ Tufts Medical Center, Boston, MA, USA; ${ }^{5}$ Brigham and Women's Hospital, Boston, MA, USA; ${ }^{6}$ Harvard Medical School, Boston, MA, USA; ${ }^{7}$ Centers for Medicare \& Medicaid Services, Baltimore, MD, USA; ${ }^{8}$ RAND Corporation, Santa Monica, CA, USA; ${ }^{9}$ David Geffen School of Medicine, Los Angeles, CA, USA.

BACKGROUND: Patient-centered medical home (PCMH) models of primary care have the potential to expand access, improve population health, and lower costs. Federally qualified health centers (FGHCs) were early adopters of PCMH models.

OBJECTIVE: We measured PCMH capabilities in a diverse nationwide sample of FQHCs and assessed the relationship between PCMH capabilities and Medicare beneficiary outcomes.

DESIGN: Cross-sectional, propensity score-weighted, multivariable regression analysis.

PARTICIPANTS: A convenience sample of 804 FQHC sites that applied to a nationwide FQHC PCMH initiative and 231,163 Medicare fee-for-service beneficiaries who received a plurality of their primary care services from these sites.

MAIN MEASURES: PCMH capabilities were self-reported using the National Committee for Quality Assurance's (NCQA's) 2011 application for PCMH recognition. Measures of utilization, continuity of care, quality, and Medicare expenditures were derived from Medicare claims covering a 1-year period ending October 2011.

KEY RESULTS: Nearly $88 \%$ of sites were classified as having PCMH capabilities equivalent to NCQA Level 1, 2, or $3 \mathrm{PCMH}$ recognition. These more advanced sites were associated with 228 additional FQHC visits per 1000 Medicare beneficiaries (95\% CI: 176, 278), compared with less advanced sites; 0.02 points higher practice-level continuity of care (95\% CI: 0.01, 0.03); and a greater likelihood of administering two of four recommended diabetes tests. However, more advanced sites were also associated with 181 additional visits to specialists per 1000 beneficiaries (95\% CI: 124, 232) and 64 additional visits to emergency departments (95\% CI: 35, 89)-but with no differences in inpatient utilization. More advanced sites had higher Part B expenditures (\$111 per beneficiary

Electronic supplementary material The online version of this article (doi:10.1007/s11606-017-4078-y) contains supplementary material, which is available to authorized users.

Received November 7, 2016

Revised April 6, 2017

Accepted April 13, 2017

Published online May 26, 2017
[95\% CI: \$61, \$158]) and total Medicare expenditures of \$353 [95\% CI: \$65, \$614]).

CONCLUSIONS: Implementation of PCMH models in FQHCs may be associated with improved primary care for Medicare beneficiaries. Expanded access to care, in combination with slower development of key PCMH capabilities, may explain higher Medicare expenditures and other types of utilization.

KEY WORDS: patient-centered medical home; federally qualified health center; quality of care; Medicare; dual eligible.

$\mathrm{J}$ Gen Intern Med 32(9):997-1004

DOI: $10.1007 / \mathrm{s} 11606-017-4078-\mathrm{y}$

c) Society of General Internal Medicine 2017

\section{INTRODUCTION}

The patient-centered medical home (PCMH) has been heralded as a model for expanding access to primary care and enhancing coordination between patients and their care teams in a way that improves population health and patient experiences while lowering cost. ${ }^{1}$ However, in several recent large multi-payer initiatives, implementation of medical homes did not reduce acute care utilization or overall expenditures. 2,3

Federally qualified health centers (FQHCs) are a subset of non-profit safety net clinics that provide comprehensive primary health care and supportive services in medically underserved areas. Even before the recent trend toward formal $\mathrm{PCMH}$ recognition, FQHCs were implementing infrastructure and practices consistent with the medical home model by adopting electronic health records (EHRs) and providing team-based and patient-centered care - often at levels exceeding those in non-FQHC primary care practices. ${ }^{4-8}$ However, achieving formal $\mathrm{PCMH}$ recognition requires $\mathrm{FQHC}$ to develop a wide range of advanced capabilities, including greater access after normal business hours, greater coordination with hospitals, and more advanced use of health information technology (IT) systems, for which FQHCs may lack staff or resources to make the necessary investments. ${ }^{9}$ Delayed development of such capabilities could affect an FQHC's ability to achieve higher-quality care and lower cost. 
A limited number of studies have examined the relationship between PCMH recognition and patient outcomes in FQHCs. Clarke et al. found that there was no significant relationship between an overall measure of PCMH implementation and the quality of care provided to patients with diabetes in a sample of California FQHCs. ${ }^{10}$ However, in a more recent analysis among FQHCs nationally, Shi et al. showed that $\mathrm{PCMH}$ recognition was associated with better prevention and chronic disease management. ${ }^{11}$ While neither of these studies examined the role of specific PCMH capabilities, other studies have shown that improved access, patient-centered communication, and wholeperson orientation were related to higher performance on process measures of quality. ${ }^{12,13}$ To our knowledge, no studies have examined the association between PCMH capabilities of FQHCs and measures of utilization or expenditures.

This study uses a sample of 804 FQHC sites that applied to a nationwide PCMH initiative to examine baseline PCMH capabilities using the National Committee for Quality Assurance's (NCQA's) 2011 PCMH recognition standards. We then examine the association between PCMH capabilities and measures of utilization, quality of care, and Medicare expenditures for Medicare beneficiaries seeking care from these sites.

\section{METHODS}

Study Population. Medicare fee-for-service (FFS) beneficiaries (regardless of age) who were continuously enrolled in Parts A and B during the period 11/1/2010 to 10/31/2011, did not have end-stage renal disease, and received a plurality of their primary care services from any of $804 \mathrm{FQHC}$ sites that applied to participate in the FQHC Advanced Primary Care Practice (APCP) Demonstration in the summer of 2011 and met the Centers for Medicare \& Medicaid Services (CMS) eligibility criteria were included in the analysis. To attribute beneficiaries to practices, we counted claims for primary care visits provided by FQHCs, rural health clinics (RHCs), and other primary care practices. We then identified the practice that provided a plurality of a beneficiary's visits.

Beneficiary-Level Outcomes. We measured study outcomes using Medicare FFS claims data for the period 11/1/2010 to 10/31/2011. Measures of utilization included FQHC visits, non-FQHC primary care visits (including visits to physicians, nurse practitioners, or physician assistants who had primary care specialties and who practiced in RHCs or office settings), specialist visits (including visits to physicians, nurse practitioners, or physician assistants who had specialties other than primary care and practiced in an $\mathrm{FQHC}$, RHC, or office setting), emergency department (ED) visits (including observation stays), all-cause admissions and admissions for ambulatory care-sensitive conditions, and 30-day unplanned hospital-wide readmissions. ${ }^{14}$ Continuity of care was assessed using the Bice-Boxerman index, ${ }^{15}$ which measures dispersion in visits across providers or, separately, across practices, on a scale of 0 to 1 . Quality was assessed using five Healthcare Effectiveness Data and Information Set (HEDIS) process measures - four for diabetes patients (HbAlc testing, low-density lipoprotein cholesterol testing, retinal eye exams, and nephropathy testing within the past 12 months), as well as a composite measure of the four tests - and, for patients with ischemic vascular disease, an annual lipid test. We calculated total Medicare expenditures as the sum of all payments made per beneficiary for inpatient, skilled nursing facility, outpatient, home health, hospice, durable medical equipment, and Part B services (provided by non-institutional providers and suppliers). We also examined two specific categories of expenditures: inpatient and Part B.

PCMH Capabilities. We measured PCMH capabilities selfreported by sites using NCQA's application for PCMH recognition (using NCQA's 2011 standards; Online Appendix Table 1). These data were submitted by all applicants to the FQHC APCP Demonstration in the summer of 2011. ${ }^{16}$ NCQA confers formal PCMH recognition (Level 1, 2, or 3) based on the number of points scored across elements after review of a site's application and associated documentation. In this study, each site completed the NCQA application for the purposes of a baseline assessment only and did not provide documentation to support their responses. To assess differences in beneficiary outcomes between sites that would likely meet criteria for PCMH recognition and those that would not, we grouped sites having point totals equivalent to at least Level 1 recognition (35 points or higher) or not sufficient to meet Level 1 recognition ( $<35$ points). In a sensitivity analysis, we defined four groups based on point totals using cut points equivalent to Level 1, 2, or 3 recognition, or no equivalent recognition.

Case Mix Adjustment and Propensity Score Weighting. We adjusted all outcomes for beneficiary, site, and area characteristics using data from Medicare enrollment and claims files, other administrative databases, and the American Community Survey. We calculated propensity scores using multinomial logistic regression to predict each beneficiary's probability of being attributed to a site with each of the four levels of PCMH capabilities. We then used inverse probability of treatment weights in regression analyses to estimate the average effect of each level of PCMH capabilities on beneficiary outcomes. This approach weights each group so that the distribution of characteristics are similar across the two groups (main analysis) or among the four groups (sensitivity analysis). ${ }^{17}$ Balance assessments are included in Online Appendix Table $2 \mathrm{a}$ and $\mathrm{b}$.

Statistical Analysis. We used logistic regression to estimate effects on readmission measures, and generalized estimating equations for all other outcomes. Among the latter, we specified negative binomial distributions for utilization 
measures, Gaussian distributions for continuity of care, binomial distributions for quality measures, and gamma distributions for expenditure outcomes (to account for the right-skewed distribution of expenditures). ${ }^{18}$ For utilization and expenditure measures, we used two-part models to account for beneficiaries with no visits or no expenditures. ${ }^{19}$ To facilitate interpretation of the results of non-linear models, we used the method of predictive margins, or "recycled predictions," 20,21 to re-transform estimates to their original measurement scales. This approach generates four sets of model predictions under four counterfactual assumptions: that all beneficiaries receive care from sites with each level of PCMH capabilities. Mean outcomes are estimated under each scenario, as well as differences between levels. We accounted for clustering of observations within sites using the Huber-White "sandwich" estimator. ${ }^{22}$ For two-part models, we used a bootstrapping procedure with 1000 replications to estimate confidence intervals (CIs).
RAND's Human Subjects Protection Committee approved the study protocol.

\section{RESULTS}

The study population included 231,163 Medicare FFS beneficiaries who received the plurality of their primary care visits between November 2010 and October 2011 from any of 804 FQHC sites that applied to participate in the FQHC APCP Demonstration. Approximately $40 \%$ of the cohort was under 65 years of age, nearly half was dually eligible for Medicare and Medicaid, and just under 30\% was either non-white or Hispanic (Table 1). As expected, given the age and high rates of dual eligibility of the cohort, comorbidities were prevalent.

Among the 804 sites included in the analysis, the vast majority were affiliated with multi-site FQHCs, and two-

Table 1 Characteristics of Medicare Beneficiaries Receiving Care from FQHCs in the Study Sample

\begin{tabular}{|c|c|c|c|}
\hline Characteristic & All Sites $(N=804)$ & $\begin{array}{l}\text { No Equivalent Recognition } \\
\text { Sites }(N=97)\end{array}$ & $\begin{array}{l}\text { Level 1, 2, or } 3 \text { Equivalent } \\
\text { Recognition Sites }(N=707)\end{array}$ \\
\hline \multicolumn{4}{|l|}{ Age (years), $n(\%)$ : } \\
\hline $18-44$ & $21,792(9.4)$ & $2193(8.1)$ & $19,599(9.6)$ \\
\hline $45-64$ & $66,609(28.8)$ & $7097(26.1)$ & $59,512(29.2)$ \\
\hline $65-74$ & $84,136(36.4)$ & $10,195(37.5)$ & $73,941(36.2)$ \\
\hline $75-84$ & $44,079(19.1)$ & $5823(21.4)$ & $38,256(18.8)$ \\
\hline 85 and older & $14,547(6.3)$ & $1863(6.9)$ & $12,684(6.2)$ \\
\hline \multicolumn{4}{|l|}{ Race/ethnicity, $n(\%)$ : } \\
\hline White & $164,073(71.0)$ & $16,974(62.5)$ & $147,099(72.1)$ \\
\hline Black & $40,456(17.5)$ & $6210(22.9)$ & $34,246(16.8)$ \\
\hline Asian & $7750(3.4)$ & $2396(8.8)$ & $5354(2.6)$ \\
\hline Hispanic & $12,961(5.6)$ & $1087(4.0)$ & $11,874(5.8)$ \\
\hline North American Native & $2299(1.0)$ & $97(0.4)$ & $2202(1.1)$ \\
\hline Other/unknown & 3624 (1.6) & $407(1.5)$ & 3217 (1.6) \\
\hline \multicolumn{4}{|l|}{ Gender, $n(\%)$ : } \\
\hline Male & $99,506(43.0)$ & $11,719(43.1)$ & $87,787(43.0)$ \\
\hline Female & $131,657(57.0)$ & $15,452(56.9)$ & $116,205(57.0)$ \\
\hline Dual eligible, $n(\%)$ & $111,449(48.2)$ & $13,039(48.0)$ & $98,410(48.2)$ \\
\hline Disabled, $n(\%)$ & $112,429(48.6)$ & $11,938(43.9)$ & $100,491(49.3)$ \\
\hline Institutionalized, $n(\%)$ & $4436(1.9)$ & $519(1.9)$ & 3917 (1.9) \\
\hline \multicolumn{4}{|l|}{ Comorbidities, $n(\%)$ : } \\
\hline Diabetes & $84,562(36.6)$ & 9928 (36.5) & $74,634(36.6)$ \\
\hline Chronic lung disorders & $41,083(17.8)$ & $4559(16.8)$ & $36,524(17.9)$ \\
\hline Chronic/disabling mental health conditions & $33,127(14.3)$ & $3324(12.2)$ & $29,803(14.6)$ \\
\hline Cardiovascular disorders & $34,968(15.1)$ & $3969(14.6)$ & $30,999(15.2)$ \\
\hline Chronic heart failure & $31,247(13.5)$ & $3544(13.0)$ & $27,703(13.6)$ \\
\hline Neurological disorders & $28,949(12.5)$ & $3055(11.2)$ & $25,894(12.7)$ \\
\hline Vascular disorders & $26,516(11.5)$ & $2881(10.6)$ & $23,635(11.6)$ \\
\hline Renal failure & $27,116(11.7)$ & $3381(12.4)$ & $23,735(11.6)$ \\
\hline Cancer & $21,525(9.3)$ & $2644(9.7)$ & $18,881(9.3)$ \\
\hline Trauma & $12.494(5.4)$ & $1384(5.1)$ & $11.110(5.4)$ \\
\hline Stroke & $10,829(4.7)$ & $1306(4.8)$ & $9523(4.7)$ \\
\hline Autoimmune disorders & $10,423(4.5)$ & $1164(4.3)$ & $9259(4.5)$ \\
\hline Chronic alcohol/drug dependence & $8206(3.5)$ & $725(2.7)$ & $7481(3.7)$ \\
\hline Infections & $6844(3.0)$ & $861(3.2)$ & $5983(2.9)$ \\
\hline Decubitus ulcer & $7019(3.0)$ & $816(3.0)$ & $6203(3.0)$ \\
\hline Moderate or end-stage liver disease & $6101(2.6)$ & $723(2.7)$ & $5378(2.6)$ \\
\hline Gastrointestinal disorders & $5897(2.6)$ & $664(2.4)$ & $5233(2.6)$ \\
\hline Protein-calorie malnutrition & 3665 (1.6) & 509 (1.9) & $3156(1.5)$ \\
\hline Pancreatic disease & 3265 (1.4) & $352(1.3)$ & $2913(1.4)$ \\
\hline HIV/AIDS & $2654(1.1)$ & $316(1.2)$ & $2338(1.1)$ \\
\hline Bone/joint/muscle infections or necrosis & $1993(0.9)$ & $217(0.8)$ & $1776(0.9)$ \\
\hline Severe hematological disorders & $1889(0.8)$ & $195(0.7)$ & $1694(0.8)$ \\
\hline
\end{tabular}

Note: "Institutionalized" was defined as having two or more skilled nursing facility stays in the previous 24 months. Comorbidities were measured using Hierarchical Condition Categories. ${ }^{23}$ Cancer comorbidity excludes pre-cancer or in situ status. The results displayed in the table are not propensity score-weighted 
Table 2 Characteristics of 804 FQHC Sites Applying to the FQHC Advanced Primary Care Practice Demonstration

\begin{tabular}{|c|c|c|c|}
\hline Characteristic & $\begin{array}{l}\text { All Sites } \\
(N=804)\end{array}$ & $\begin{array}{l}\text { No Equivalent } \\
\text { Recognition Sites } \\
(N=97)\end{array}$ & $\begin{array}{l}\text { Level 1, 2, or } 3 \\
\text { Equivalent Recognition } \\
\text { Sites }(N=707)\end{array}$ \\
\hline \multicolumn{4}{|c|}{ PCMH capabilities (NCQA 2011 PCMH recognition level equivalent scores), $n(\%)$ : } \\
\hline No recognition equivalent ( $<35$ points) & $97(12.1)$ & $97(100.0)$ & \\
\hline Level 1 equivalent ( $35-59$ points) & $304(37.8)$ & & $304(43.0)$ \\
\hline Level 2 equivalent ( $60-85$ points) & $324(40.3)$ & & $324(45.8)$ \\
\hline Level 3 equivalent ( $\geq 85$ points) & $79(9.8)$ & & $79(11.2)$ \\
\hline \multicolumn{4}{|l|}{ Number of FQHC service delivery sites, $n(\%)$ : } \\
\hline 1 & $23(2.9)$ & $2(2.1)$ & $21(3.0)$ \\
\hline $2-10$ & $439(54.7)$ & $43(44.3)$ & $396(56.1)$ \\
\hline $11+$ & $341(42.5)$ & $52(53.6)$ & $289(40.9)$ \\
\hline \multicolumn{4}{|l|}{ Years in operation, $n(\%)$ : } \\
\hline $1-9$ & $259(32.2)$ & $31(32.0)$ & $228(32.2)$ \\
\hline $10-19$ & $216(26.9)$ & $28(28.9)$ & $188(26.6)$ \\
\hline $20-29$ & 109 (13.6) & $10(10.3)$ & $99(14.0)$ \\
\hline $30-39$ & 148 (18.4) & $12(12.4)$ & $136(19.2)$ \\
\hline $40+$ & $49(6.1)$ & $8(8.2)$ & $41(5.8)$ \\
\hline Missing & $23(2.9)$ & $8(8.2)$ & $15(2.1)$ \\
\hline \multicolumn{4}{|l|}{ Geographic region, $n(\%)$ : } \\
\hline Central & $194(24.1)$ & $21(21.6)$ & $173(24.5)$ \\
\hline Mid-Atlantic & 118 (14.7) & $34(35.1)$ & $84(11.9)$ \\
\hline Northeast & 96 (11.9) & $2(2.1)$ & $94(13.3)$ \\
\hline Southeast & $121(15.0)$ & $12(12.4)$ & $109(15.4)$ \\
\hline West & 139 (17.3) & 14 (14.4) & $125(17.7)$ \\
\hline West-Central & $136(16.9)$ & $14(14.4)$ & $122(17.3)$ \\
\hline \multicolumn{4}{|l|}{ Metropolitan status, $n(\%)$ : } \\
\hline Metropolitan area & $548(68.2)$ & $74(76.3)$ & $474(67.0)$ \\
\hline Non-metropolitan urban area & $155(19.3)$ & $18(18.6)$ & $137(19.4)$ \\
\hline Non-metropolitan rural area & $101(12.6)$ & $5(5.2)$ & $96(13.6)$ \\
\hline Receiving PCMH payments from one or more plans, $n(\%)$ & $81(10.1)$ & $11(11.3)$ & $70(9.9)$ \\
\hline \multicolumn{4}{|l|}{ Payer mix, mean (SD) } \\
\hline Commercial insurance & $18.1(12.9)$ & $18.3(13.3)$ & $18.1(12.9)$ \\
\hline Medicaid & $34.1(15.1)$ & $33.3(16.7)$ & $34.2(14.8)$ \\
\hline Medicare (includes dual-eligibles) & $15.1(9.9)$ & $15.1(10.9)$ & $15.1(9.8)$ \\
\hline No insurance & $27.1(15.6)$ & $26.3(15.8)$ & $27.2(15.6)$ \\
\hline Other & $0.6(0.5)$ & $0.7(0.5)$ & $0.6(0.5)$ \\
\hline
\end{tabular}

Notes: NCQA = National Committee for Quality Assurance; $P C M H=$ patient-centered medical home. PCMH capabilities were assessed using NCQA's 2011 application for PCMH recognition, which awards points for meeting each of NCQA's recognition standards. We used point totals to define four levels of PCMH capabilities equivalent to each of NCQA's PCMH recognition levels (or no recognition). Because each site's responses did not undergo review by NCQA auditors, these categories do not necessarily reflect a site's true recognition level. The results displayed in the table are not propensity score-weighted

thirds had been operating for 10 years or longer (Table 2). The sites were well distributed across the United States; were located in metropolitan, urban, and rural areas; and were most likely to provide services to patients who had Medicaid coverage or had no insurance (34 and $27 \%$ of their patient populations, respectively).

The $804 \mathrm{FQHCs}$ varied widely in the extent to which they had adopted components of the PCMH model at the time of the assessment. While no FQHCs had received formal PCMH recognition according to NCQA's 2011 standards, 79 sites (almost 10\%) had self-reported PCMH capabilities that were equivalent to Level 3 recognition, while 40 and $38 \%$ had the equivalent of Level 2 and Level 1 recognition, respectively (Table 2). Only $10 \%$ of sites reported receiving incentive payments from health plans because of their status as a PCMH based on NCQA's 2008 recognition program or another program.

Sites with more advanced PCMH capabilities reported higher scores than less advanced sites across each of the six NCQA 2011 PCMH standards and each of their 28 constituent "elements" (Table 3). Differences between groups ranged from 38 to 48 percentage points across the six standards and were larger for several elements. The largest differences in element scores were for coordination with facilities (Element $5 \mathrm{C} ; 59$ percentage points) and performance measurement and quality improvement capabilities (Elements 6A, 6C, and 6D; 53-56 percentage points). Other large differences included foundational aspects of the PCMH model, such as establishing practice-level processes that enhance access and coordination (Element 1E; 56 percentage points) and organizing and training members of patient care teams (Element 1G; 53 percentage points).

Low levels of capabilities in several elements across both groups were also notable. For example, more advanced sites also reported limited ability to identify high-risk patients (Element 3B; 34 percentage points), optimize care management processes (Element $3 \mathrm{C} ; 37$ percentage points), and support patients' self-care processes (Element 4A; 38 percentage points). Online Appendix Table 3 displays summaries of PCMH capabilities stratified by recognition level.

Associations between PCMH capabilities and beneficiary utilization, continuity of care, process measures, and Medicare 
Table 3 Differences in Self-Reported PCMH Capabilities for Sites With NCQA Level 1, Level 2, or Level 3 Equivalent PCMH Recognition and Sites With No Equivalent Recognition

\begin{tabular}{|c|c|c|c|}
\hline & \multicolumn{2}{|c|}{$\begin{array}{l}\text { Score (\% of available points } \\
\text { achieved) }\end{array}$} & \multirow{2}{*}{$\begin{array}{l}\text { Difference (Level 1, 2, } \\
\text { or } 3 \text { Equivalent } \\
\text { Recognition - No } \\
\text { Equivalent Recognition) } \\
\text { (percentage points) }\end{array}$} \\
\hline & $\begin{array}{l}\text { No Equivalent } \\
\text { Recognition } \\
\text { Sites }(N=97)\end{array}$ & $\begin{array}{l}\text { Level 1, 2, or } \\
3 \text { Equivalent } \\
\text { Recognition } \\
\text { Sites }(N=707)\end{array}$ & \\
\hline 1: Enhance Access and Continuity $(\mathrm{A}-\mathrm{G})$ & 21.5 & 61.1 & 39.6 \\
\hline A - Access during office hours & 23.0 & 64.2 & 41.2 \\
\hline D - Continuity & 18.4 & 65.9 & 47.5 \\
\hline E - Medical home responsibilities & 10.9 & 66.4 & 55.6 \\
\hline F - Culturally appropriate services & 66.4 & 97.8 & 31.4 \\
\hline $\mathrm{G}$ - The practice team & 7.9 & 61.1 & 53.2 \\
\hline 2: Identify and Manage Patient Populations (A-D) & 35.1 & 72.6 & 37.5 \\
\hline A - Patient information & 78.0 & 96.9 & 19.0 \\
\hline B - Clinical data & 22.4 & 69.6 & 47.2 \\
\hline $\mathrm{C}$ - Care management & 2.6 & 37.0 & 34.3 \\
\hline D - Medication management & 14.5 & 61.7 & 47.2 \\
\hline E - Use electronic prescribing & 31.6 & 58.9 & 27.3 \\
\hline 4: Provide Self-Care Support and Community Resources (A, B) & 15.1 & $\mathbf{5 2 . 7}$ & 37.5 \\
\hline A - Support self-care process & 4.6 & 37.7 & 33.1 \\
\hline $\mathrm{B}-$ Referrals to community resources & 36.2 & 82.7 & 46.5 \\
\hline 5: Track and Coordinate Care (A-C) & 19.3 & 64.3 & 45.0 \\
\hline A - Test tracking and follow-up & 22.4 & 55.5 & 33.1 \\
\hline B - Referral tracking and follow-up & 31.3 & 74.3 & 43.1 \\
\hline $\mathrm{C}-$ Coordinate with facilities/transitions & 4.3 & 63.0 & 58.7 \\
\hline 6: Measure and Improve Performance $(\mathrm{A}-\mathrm{G})$ & 28.5 & 76.8 & 48.2 \\
\hline A - Measure performance & 24.7 & 78.0 & 53.3 \\
\hline B - Measure patient/family experience & 27.3 & 64.2 & 36.9 \\
\hline C - Implement continuous QI & 25.3 & 81.0 & 55.7 \\
\hline D - Demonstrate continuous QI & 34.2 & 89.1 & 54.9 \\
\hline
\end{tabular}

$Q I=$ quality improvement

Note: P values for differences between sites with no recognition and sites with Level 1, Level 2, or Level 3 equivalent recognition were all less than 0.001. Data were from the National Committee for Quality Assurance's 2011 application for PCMH Recognition, completed by 804 FQHC sites and submitted as part of their application to the CMS Federally Qualified Health Center Advanced Primary Care Practice Demonstration in the summer of 2011. No recognition was defined as a score of 0-34 points; Level 1/2/3-equivalent recognition was defined as 35-100 points

expenditures were mixed (Table 4). More advanced sites had higher rates of FQHC visits (228 additional visits per 1000 beneficiaries; 95\% CI [176, 278]) than less advanced sites. More advanced sites also had higher utilization rates for specialist services (181 additional specialist visits per 1000 beneficiaries; 95\% CI $[124,232])$ and higher rates of ED visits (64 additional ED visits per 1000 beneficiaries; 95\% CI [35, 89]). Among inpatient measures, we observed no association between PCMH capabilities and all-cause admissions, admissions for ambulatory care-sensitive conditions, or readmissions.

Provider-level continuity of care was worse among sites with greater PCMH capabilities $(0.03$ points lower on the 0 -to- 1 scale of the Bice-Boxerman index $(95 \%$ CI $[-0.04,-0.02])$. By contrast, practice-level continuity was better among more advanced sites ( 0.02 points higher, $95 \%$ CI [0.01, 0.03]).

We found a strong positive association between PCMH capabilities and performance on two of four process measures of quality for diabetes. Rates of LDL cholesterol testing and nephropathy testing were substantially higher in more advanced sites than in less advanced sites: LDL testing rates were 2.8 percentage points higher $(95 \%$ CI $[0.4,5.1])$ and nephropathy testing rates were 7.7 percentage points higher (95\% CI [4.1, 11.3]). In addition, more advanced sites were 4.6 percentage points more likely to provide all four recommended diabetes tests (95\% CI $[2.5,6.7]$ ).

Lastly, total Medicare expenditures per beneficiary were $\$ 353$ higher, on average, for more advanced sites (95\% CI [\$65, \$614]). These differences appear to be driven by higher Part B expenditures in sites with higher levels of PCMH capabilities (\$111 higher, 95\% CI $[\$ 61, \$ 158])$.

When examining the relationship for each of three levels of NCQA-equivalent recognition separately, relative to the least advanced sites, we did not observe a strong association 
Table 4 Effect of Level 1, Level 2, or Level 3 Equivalent PCMH Recognition on Utilization, Continuity of Care, Process Measures, and Medicare Expenditures

\begin{tabular}{|c|c|c|c|c|}
\hline & \multicolumn{2}{|l|}{ Estimate } & \multirow[b]{2}{*}{$\begin{array}{l}\text { Difference (Level 1, 2, } \\
\text { or } 3 \text { Equivalent } \\
\text { Recognition - No } \\
\text { Equivalent } \\
\text { Recognition) }\end{array}$} & \multirow[b]{2}{*}{$\begin{array}{l}95 \% \\
\text { Confidence } \\
\text { Interval }\end{array}$} \\
\hline & $\begin{array}{l}\text { No Equivalent } \\
\text { Recognition } \\
\text { Sites }(N=97)\end{array}$ & $\begin{array}{l}\text { Level 1, 2, or } \\
3 \text { Equivalent } \\
\text { Recognition } \\
\text { Sites } \\
(N=707)\end{array}$ & & \\
\hline \multicolumn{5}{|l|}{ Utilization, per 1000 beneficiaries per year } \\
\hline FQHC visits & 4705 & 4933 & 228 & 176,278 \\
\hline Non-FQHC primary care visits & 417 & 414 & -3 & $-19,11$ \\
\hline Specialist visits & 2993 & 3173 & 181 & 124,232 \\
\hline ED visits & 942 & 1007 & 64 & 35,89 \\
\hline Inpatient admissions & 346 & 340 & -6 & $-22,6$ \\
\hline Inpatient $\mathrm{ACSC}$ admissions & 44 & 47 & 3 & $-2,7$ \\
\hline Inpatient readmissions, percentage points & 14.2 & 14.8 & 0.6 & $-0.5,1.6$ \\
\hline \multicolumn{5}{|l|}{ Continuity of Care, points } \\
\hline Provider-level continuity & 0.69 & 0.66 & -0.03 & $-0.04,-0.02$ \\
\hline Practice-level continuity & 0.83 & 0.85 & 0.02 & $0.01,0.03$ \\
\hline \multicolumn{5}{|l|}{ Process, percentage points } \\
\hline All four recommended diabetes tests & 20.6 & 25.2 & 4.6 & $2.5,6.7$ \\
\hline HbA1c test & 84.3 & 86.4 & 2.1 & $-1.1,5.3$ \\
\hline LDL test & 78.1 & 80.8 & 2.8 & $0.4,5.1$ \\
\hline Eye exam & 43.5 & 44.3 & 0.8 & $-1.9,3.6$ \\
\hline Nephropathy test & 50.5 & 58.2 & 7.7 & 4.1, 11.3 \\
\hline $\begin{array}{l}\text { Lipid test for patients with ischemic vascular } \\
\text { disease }\end{array}$ & 76.1 & 77.4 & 1.3 & $-1.1,3.8$ \\
\hline \multicolumn{5}{|c|}{ Medicare expenditures, dollars per beneficiary per year } \\
\hline Total Medicare expenditures & 9458 & 9811 & 353 & 65,614 \\
\hline Inpatient expenditures & 3256 & 3214 & -42 & $-198,96$ \\
\hline Part B expenditures & 1813 & 1924 & 111 & 61,158 \\
\hline
\end{tabular}

HbAlc $=$ glycated hemoglobin, $L D L=$ low-density lipoprotein cholesterol

Note: Bold entries denote differences that are statistically different from zero based on a P value $<0.05$. All models incorporate propensity score weights and are adjusted for beneficiary characteristics (age, race, gender, dual eligibility, disability, institutionalization, Hierarchical Condition Category score); site characteristics (revenue, years in operation, number of primary care providers, number of specialists, number of other affiliated sites, ambulatory care accreditation, participation in a Health Center Controlled Network, \% uninsured patients, \% Medicaid patients, FQHC Affordable Care Act grant recipient); and area characteristics (rural-urban continuum code, \% household poverty in the site's census tract). A total of 8276 of 239,439 observations (3.5\%) were dropped from the main analysis because the propensity scores for these beneficiaries fell outside of the common support (i.e., did not overlap with those in the alternative group).

$F Q H C$ visits include any visit to an FQHC regardless of provider specialty. Non-FQHC primary care visits include visits to primary care physicians, nurse practitioners, and physician assistants who practice in rural health clinics or office settings. Specialist visits include visits to physicians, nurse practitioners, or physician assistants who have specialties other than primary care and who practice in an FQHC, rural health clinic, or office setting. $E D$ visits include both ED visits that did and did not lead to a hospitalization, as well as observation stays. Inpatient ACSC [ambulatory care-sensitive condition] admissions are those with a primary diagnosis of: one or more diabetes short-term complications; one or more diabetes long-term complications; chronic obstructive pulmonary disease or asthma in older adults; hypertension; congestive heart failure; angina without procedure; uncontrolled diabetes; asthma in younger adults; and lower-extremity amputation among patients with diabetes. Inpatient readmissions were measured as 30-day unplanned hospital-wide readmissions.

Total Medicare expenditures include inpatient, outpatient, skilled nursing facility, home health, hospice, durable medical equipment, and Part B expenditures. Inpatient expenditures include all claims found in the inpatient file. Part B expenditures include all claims for services found in the carrier file (also known as the Physician/Supplier Part B claims file).

between recognition levels and outcomes (Online Appendix Table 4). Level 3 equivalent sites differed from both Level 1 and Level 2 equivalent sites in that they had lower rates of inpatient admissions and inpatient expenditures, lower rates of non-FQHC primary care visits, and no statistically significant differences in ED visits or Part B expenditures. In general, however, Level 1, Level 2, and Level 3 equivalent sites exhibited few differences across measures.

\section{DISCUSSION}

We conducted a cross-sectional analysis of a convenience sample of $804 \mathrm{FQHC}$ sites to assess self-reported PCMH capabilities prior to the initiation of a nationwide demonstration, and to assess the relationship between these PCMH capabilities and the care and outcomes of Medicare beneficiaries. Overall, our findings were mixed: although sites with greater PCMH capabilities tended to have higher rates of FQHC visits, higher quality of care, and greater continuity of care (on one of two measures), these sites were also associated with higher rates of visits to EDs and specialists and higher Part B and total expenditures.

Higher rates of $\mathrm{FQHC}$ visits in more advanced sites may be the result of these sites' efforts to expand access to care both during and after normal business hours or may reflect well-functioning practice teams that are able to expand the number of appointments available to patients. Enhanced access may also explain the positive association we observed between PCMH capabilities and 
quality of care for patients with diabetes-findings that stand in contrast to those of a previous study that found little association between PCMH capabilities and the quality of diabetes care using a smaller, more homogeneous sample of FQHCs and patients. ${ }^{10}$ Use of patient care strategies such as standing orders and more robust population health management strategies among sites with more advanced PCMH capabilities may also explain these results.

Our findings of higher levels of Medicare expenditures and greater ED and specialty care utilization associated with more advanced PCMHs have several possible explanations. More advanced PCMHs may be addressing unmet specialty care needs for their most vulnerable populations. ${ }^{24,}{ }^{25}$ Prior studies also suggest that FQHCs may tell their most vulnerable patients who cannot wait for appointments to seek care in the ED. ${ }^{26}$ It is also possible that reductions in inpatient utilization and readmission measures require PCMH capabilities that are not yet available in most FQHCs. For example, identifying high-risk patients and optimizing care management systems, which includes developing care plans and identifying and addressing barriers to achieving patients' treatment goals, are two areas in which sites were least likely to meet NCQA's standards. Further development of these capabilities might help FQHCs achieve reductions in utilization and expenditures.

Our mixed findings in terms of continuity of care, in which continuity with the same provider was lower, but continuity with the same practice was higher, in sites with greater PCMH capabilities indicate that these sites are more likely to provide care to patients consistently over time, even if the patients do not always receive care from the same provider. Such a pattern is expected when practices implement team-based care or expand same-day access. Nevertheless, the magnitude of the differences between more advanced and less advanced sites is small. Other studies commonly report clinically meaningful effects on patient outcomes when between-group differences in continuity exceed 0.1 point on the index's $0-1$ scale. $^{27}$

We found limited evidence of larger effects on patient outcomes associated with increasing levels of PCMH capabilities. One possible explanation is that sites may have under- or overstated their PCMH capabilities because of lack of familiarity with NCQA's updated (2011) PCMH recognition standards, which were released several months before the assessment. Measurement error associated with PCMH capabilities might have led to the misclassification of sites' recognition levels, which could have biased the results of these analyses toward finding no association. At the same time, it is possible that the NCQA PCMH instrument may not be optimally designed to detect these associations. Our analyses used the 2011 recognition standards; however, the instrument has continued to evolve over time in response to stakeholder feedback.
Our study has several important limitations. First, the crosssectional, observational study design does not allow for inferences about causality. Only longitudinal analyses assessing changes in outcomes following PCMH recognition can address the question of whether PCMH implementation may reduce specialty care and acute care utilization and expenditures over time. Second, although we adjusted for a large number of observable beneficiary, site, and area characteristics, and we used propensity score-weighting techniques consistent with best practices ${ }^{28}$ differences in unobserved characteristics such as clinic leadership and access to resources could lead to spurious associations between PCMH capabilities and beneficiary outcomes. Third, the analysis was limited to Medicare and dually eligible beneficiaries, which represent approximately $12 \%$ of FQHC patients nationally, ${ }^{29}$ and study outcomes were derived from Medicare claims data and do not include patient experience measures. The five process measures of quality used in the analysis were selected because of the high prevalence of these conditions among Medicare beneficiaries, and because each screening test is recommended annually and is thus likely to be sensitive to PCMH-related practice changes. Using additional process measures could provide a more comprehensive assessment. Fourth, data on PCMH capabilities were collected using a self-reported, Web-based survey tool that could include measurement error. Lastly, the 804 sites included in our study were drawn from a convenience sample of applicants to a PCMH initiative and may not be representative of typical FQHC sites across the United States. Furthermore, a small percentage of beneficiaries were dropped from the analysis to improve the comparability of sites, which may also lessen generalizability.

The associations found between PCMH capabilities and increased $\mathrm{FQHC}$ visits, increased delivery of evidence-based care for patients with diabetes, and higher continuity of care with FQHC sites suggest that PCMHs may have beneficial impacts on Medicare beneficiaries who receive care at FQHCs. These findings provide some support for the efforts of payers and policymakers to encourage implementation of PCMHs within FQHCs. However, our study also suggests that certain PCMH capabilities, including identifying high-risk patients, effectively using care plans, and supporting self-care, may be particularly difficult for FQHCs to develop despite their overall high level of capabilities. Practice transformation efforts involving FQHCs may need to consider strategies to achieve these PCMH capabilities to help realize the anticipated reductions in utilization and expenditures over time.

Acknowledgements: The authors thank the participants in the Federally Qualified Health Center Advanced Primary Care Practice Demonstration, Lisa Parker from the Center for Medicare and Medicaid Innovation for feedback on earlier versions of this manuscript, and Monique Martineau from RAND for editorial assistance. The analyses upon which this publication is based were performed under Contract No. HHSM-500-2005-00028I/Task Order HHSM-500T0008, entitled "Evaluation of the Federally Qualified Health Center 
Advanced Primary Care Practice Demonstration," funded by the Centers for Medicare \& Medicaid Services, Department of Health and Human Services. The content of this publication does not necessarily reflect the views or policies of the Department of Health and Human Services, nor does the mention of trade names, commercial products, or organizations imply endorsement by the U.S. government. The authors assume full responsibility for the accuracy and completeness of the ideas presented.

Preliminary results from this study were presented at the 2014 AcademyHealth Annual Research Meeting in San Diego, CA.

Corresponding Author: Justin W. Timbie, PhD; RAND Corporation, Arlington, VA, USA (e-mail: jtimbie@rand.org).

\section{Compliance with Ethical Standards:}

Conflict of Interest: The authors declare that they do not have a conflict of interest.

\section{REFERENCES}

1. American Academy of Family Physicians, American College of Physicians, American Osteopathic Association. Joint principles of the patient-centered medical home, 2007. http://www.aafp.org/ dam/AAFP/documents/practice_management/pcmh/initiatives / PCMHJoint.pdf. Accessed 4/10/17.

2. Dale SB, Ghosh A, Peikes DN, Day TJ, Yoon FB, Taylor EF, et al. TwoYear Costs and Quality in the Comprehensive Primary Care Initiative. N Eng J Med. 2016;374(24):2345-56.

3. Friedberg MW, Schneider EC, Rosenthal MB, Volpp KG, Werner RM. Association between participation in a multipayer medical home intervention and changes in quality, utilization, and costs of care. JAMA. 2014;311(8):815-25.

4. Goldman LE, Chu PW, Tran H, Romano MJ, Stafford RS. Federally qualified health centers and private practice performance on ambulatory care measures. Am J Prev Med. 2012;43(2):142-9.

5. Michelle M. Doty, Melinda K. Abrams, Susan E. Hernandez, Kristof Stremikis, Beal AC. Enhancing the Capacity of Community Health Centers to Achieve High Performance. New York: The Commonwealth Fund, 2010.

6. Jones EB, Furukawa MF. Adoption and use of electronic health records among federally qualified health centers grew substantially during 201012. Health Aff (Millwood). 2014;33(7):1254-61.

7. Rothkopf J, Brookler K, Wadhwa S, Sajovetz M. Medicaid patients seen at federally qualified health centers use hospital services less than those seen by private providers. Health Aff (Millwood). 2011;30(7):1335-42.
8. Wright B, Potter AJ, Trivedi A. Federally qualified health center use among dual eligibles: Rates of hospitalizations and emergency department visits. Health Aff (Millwood). 2015;34(7):1147-55.

9. Guinn MT, Gunter KE, Nocon RS, Lewis SE, Vable AM, Tang H, et al. Undergoing transformation to the patient centered medical home in safety net health centers: perspectives from the front lines. Ethn Dis. 2013;23(3):356-62.

10. Clarke RM, Tseng CH, Brook RH, Brown AF. Tool used to assess how well community health centers function as medical homes may be flawed. Health Aff. 2012;31(3):627-35.

11. Shi L, Lee DC, Chung M, Liang H, Lock D, Sripipatana A. Patientcentered medical home recognition and clinical performance in U.S. community health centers. Health Serv Res. 2016.

12. Ferrante JM, Balasubramanian BA, Hudson SV, Crabtree BF. Principles of the patient-centered medical home and preventive services delivery. Ann Fam Med. 2010;8(2):108-16.

13. Lebrun-Harris LA, Shi L, Zhu J, Burke MT, Sripipatana A, NgoMetzger Q. Effects of patient-centered medical home attributes on patients' perceptions of quality in federally supported health centers. Ann Fam Med. 2013;11(6):508-16.

14. Horwitz L, Partovian C, Lin Z, Herrin J, Grady J, Conover M, et al. Hospital-wide all-cause unplanned readmission measure: final technical report. 2012.

15. Bice TW, Boxerman SB. A quantitative measure of continuity of care. Med Care. 1977;15(4):347-9.

16. National Committee for Quality Assurance. PCMH 2011-PCMH 2014 crosswalk. Washington, DC (Undated). http://www.ncqa.org/Programs/ Recognition / Practices / PatientCenteredMedicalHomePCMH / PCMH2011PCMH2014Crosswalk.aspx. Accessed 4/10/17.

17. Austin PC. An introduction to propensity score methods for reducing the effects of confounding in observational studies. Multivar Behav Res. 2011;46(3):399-424.

18. Dodd S, Bassi A, Bodger $\mathbf{K}$, Williamson P. A comparison of multivariable regression models to analyse cost data. J Eval Clin Pract. 2006;12(1):7686.

19. Buntin MB, Zaslavsky AM. Too much ado about two-part models and transformation? Comparing methods of modeling Medicare expenditures. J Health Econ. 2004;23(3):525-42.

20. Graubard BI, Korn EL. Predictive margins with survey data. Biometrics. 1999;55(2):652-9.

21. Setodji CM, Scheuner M, Pankow JS, Blumenthal RS, Chen $\mathbf{H}$, Keeler E. A graphical method for assessing risk factor threshold values using the generalized additive model: the multi-ethnic study of atherosclerosis. Health Serv Outcomes Res Methodol. 2012;12(1):6279.

22. White $\mathbf{H}$. A heteroskedasticity-consistent covariance-matrix estimator and a direct test for heteroskedasticity. Econometrica. 1980;48(4):817-38.

23. Pope G, Kautter J, Ellis R, Ash A, Ayanian J, Iezzoni L, et al. Risk adjustment of Medicare capitation payments using the CMS-HCC model. Health Care Financ Rev. 2004;25(4):119-42. 